\title{
Design and testing of an optical configuration for multi-dimensional measurement of a diamond cutting tool
}

\author{
Yuki Shimizu*, SungHo Jang, and Wei Gao \\ Department of Nanomechanics, Tohoku University, Sendai, 980-8579, Japan \\ *Phone \& Fax: +81-22-795-6950, E-mail: yuki.shimizu@nano.mech.tohoku.ac.jp
}

\begin{abstract}
This paper presents a new optical measuring instrument for multi-dimensional measurement of a diamond cutting tool to be used in diamond turning process. The measuring instrument is composed of a micro-optical probe component for tool edge contour measurement and a Linnik microscope component for tool faces measurement. In the micro-optical probe component, a focused laser beam scans along a tool edge to measure the deviation of the tool edge contour from the scanning path of the laser beam. For further precise tool edge contour measurement with the micro-optical probe component, its spot diameter needs to be optimized. The Linnik microscope component, which is an interferometric microscope having a pair of matched objective lenses in its optical setup, is therefore integrated into the measuring instrument not only to measure tool faces but also to optimize the spot diameter of the focused laser beam in the micro-optical probe component. The optical configuration of the proposed optical measuring instrument is designed, and its prototype composed of the micro-optical probe component and the Linnik microscope component is developed based on an optical cage system. Furthermore, some basic experiments are carried out to verify the feasibility of the developed prototype.
\end{abstract}

Keywords: Measurement, Cutting tool, Edge contour measurement, Flank face, Rake face, Interferometric microscope 


\section{Introduction}

Diamond turning using diamond tools is one of ultra-precision machining technologies for fabrication of highly-accurate precision parts [1-3]. In recent, diamond tools are often used for fabricating products having three-dimensional micro-structures [4]. In the diamond turning process, surface form is fabricated on a workpiece by transferring an edge contour of a cutting tool. Since even a nanometric wear on the tool tip sometimes results in a significant surface form error [5], tool cutting edge inspection is an essential task for the assurance of the machining accuracy. In addition, for highly-accurate diamond turning, not only tool edge contour but also tool faces need to be inspected because a tool edge contour is determined as an intersection of a tool rake face and a tool flank face. The qualities of tool faces also affect roughness of machined surfaces, cutting efficiency and so on [6]. Meanwhile, measurement of such a cutting tool is preferred to be carried out in on-machine condition so that installation error due to detachment of a cutting tool from a machine tool can be avoided [7]; even though such installation error is small, it should be taken into consideration in the case of ultra-precision machining.

Scanning electron microscopes (SEMs) have often been employed for qualitative evaluation of tool edge contours [8], since SEMs have advantages of a large field of view, a high measurement resolution, non-contact imaging and so on. However, on the other hand, it costs a lot to acquire quantitative information of tool edge contours from two-dimensional SEM images. A lot of efforts have therefore been made so far to carry out quantitative tool edge contour evaluation based on SEMs [9, 10]. However, SEMs have the other disadvantages; SEMs are difficult to be applied for on-machine measurement since they require vacuum condition for measurement. Furthermore, high-energy electric beams can damage tool edges having nanometric sharpness.

Optical measuring instruments such as focus variation instruments or confocal microscopes have also been used to observe diamond cutting tools [11-13]. Features of these optical measuring instruments include non-contact, fast and non-destructive measurement. Tool wear on cutting edges can also be evaluated by using these instruments [14-15]. However, these conventional optical 
measuring instruments cannot measure nanometer-level tool edge contour. In addition, the horizontal resolution is limited by the diffraction limit of light. Although some state-of-the-art optical measuring instruments specialized for tool profile measurement are available in the market $[16,17]$, quantitative evaluation of the cutting edge of diamond cutting tool in nanometer-level is still a difficult task.

On the other hand, Atomic force microscopes (AFMs), which have several advantages such as sub-nanometric resolutions in three-dimension and low measuring force, are reasonable instruments for nanometric tool edge measurement [18]. In general, however, it is a time-consuming task to align an AFM probe with respect to a three-dimensional miniature structure such as a cutting edge of a diamond tool. In responding to this problem, an AFM-based instrument for three-dimensional tool edge measurement has been introduced with a combination of a focused laser beam, which enables a tip of the AFM probe to be aligned with respect to a tool edge [19]. A unique alignment sequence is employed so that precise tool-tip alignment can be carried out in a short time. Feasibility of the developed instrument has been verified by measuring three-dimensional tool edge profiles with a nose radius ranging from $2 \mu \mathrm{m}$ to $200 \mu \mathrm{m}$ [20]. Furthermore, a method for tool edge contour measurement by using the focused laser beam as a measuring probe, which is referred to as the micro-optical probe in this paper, has also been proposed, and its feasibility has been verified in experiments $[21,22]$.

In this paper, not only to improve the measurement accuracy of the micro-optical probe but also to achieve tool face measurement, a new concept for multi-dimensional measurement of a diamond cutting tool is proposed. As a first step for the achievement of the proposed concept, design study for an optical measuring instrument composed of a micro-optical probe component and an interferometric microscope component based on the Linnik configuration [23], which is referred to as the Linnik microscope component in this paper, is carried out. The instrument is designed in such a way that the two optical components share the same measuring optical axis. Based on the designed optical configuration, a prototype of the optical measuring instrument is developed in an 
optical cage system. To verify the feasibility of the developed prototype, some basic experiments are carried out. A detailed description of the developed prototype of the optical measuring instrument, and results of the basic experiments are presented.

\section{Principle of multi-dimensional measurement of a diamond cutting tool}

\subsection{An overview of the developed optical configuration}

A schematic of a single-point diamond cutting tool, which is simply referred to as a cutting tool in this paper, is shown in Fig. 1. A tool cutting edge contour is one of important parameters to be evaluated since its shape will directly be transferred to a machining surface. In the case of a cutting tool with a round nose, its edge contour is determined as a circular arc with a constant radius $(R)$ ranging from a few $\mu \mathrm{m}$ to several $\mathrm{mm}$. The tool edge contour is determined as an intersection of two surfaces, which are often referred to as a rake face and a flank face as shown in Fig. 1. Qualities of the rake face and flank face affect cutting force during diamond turning process and roughness of machined surfaces. Therefore, not only the tool edge contour but also the tool faces are required to be evaluated by appropriate methods. In this paper, optical configurations for both the micro-optical probe component and the Linnik microscope component are designed. The micro-optical probe component is used to measure tool edge contours with a high measurement resolution beyond the diffraction limit [21], while the Linnik microscope component is used to measure tool faces with a nanometric vertical resolution. The detail of the design of the optical configuration is described in the following subsections.

\subsection{Micro-optical probe component}

For on-machine tool edge contour measurement, the micro-optical probe has been developed [21]. In this paper, its optical setup is optimized in terms of a compatibility with the Linnik microscope component, which is described in the following subsection. Figure 2(a) shows a schematic of the optical configuration of the micro-optical probe. The micro-optical probe is 
composed of a laser diode (LD), a collimating lens, an objective lens and a photodiode (PD). A laser emitted from the LD is collimated by the collimating lens to generate a collimated laser beam. The collimated laser beam is made to pass through the objective lens so that a micro-sized laser spot can be generated on its focal plane. A cutting tool to be measured will be mounted on a positioning system, and will be aligned with respect to the micro-optical probe in such a way that a part of the micro-optical probe covers the tool rake face. The other part of the micro-optical probe passes through the focal plane, and is captured by the PD placed just beneath the cutting tool. A deviation of the light intensity of the laser beam detected by the PD corresponds to the $Y$-directional deviation $\delta$ of the center position of the laser spot with respect to the cutting edge contour. A relationship between the $Y$-position of the micro-optical probe and the light intensity of the laser beam passed through the focal plane can be verified by carrying out a calibration in advance of the tool edge contour measurement. Now the $X Y$-coordinates of the center of the micro-optical probe on its focal plane are provided by displacement sensors such as capacitive sensors or linear encoders embedded in the $X Y$-stage system. Therefore, the $X Y$-coordinates of each point on the cutting edge can be acquired from the displacement sensor outputs and $\delta$. The cutting edge contour can be acquired by scanning the micro-optical probe along the tool cutting edge. The sensitivity of the calibration curve depends on a diameter of the micro-optical probe. Therefore, a cutting tool to be measured is expected to be positioned in the focal plane of the objective lens as precise as possible. It should be noted that the calibration curve is valid until the diameter of the micro-optical probe is small compared with the tool nose radius.

In the concept of the micro-optical probe, the accuracy of the displacement sensors, which are employed in the stage system for giving tool motions in the $X Y$-directions, directly affects the accuracy of tool edge contour measurement. Meanwhile, in most of the cases, single-point diamond cutting tools are used in ultra-precision lathes employing ultra-precision positioning system with highly-accurate displacement sensors. Therefore, the micro-optical probe is suitable for on-machine measurement. In this paper, an $X Y$ stage system consisting of a pair of one-axis servo motor stages, 
in each of which a displacement sensor is embedded, is employed to give the $X Y$-directional motions to a cutting tool.

\subsection{Linnik microscope component}

Figure 2(b) shows a schematic of an optical setup for tool face measurement. The optical setup is based on the Linnik configuration, which is one of optical configurations for interferometric microscopes [23]. The optical setup consists of a light source, a beam splitter, a pair of objective lenses, a reference mirror, an imaging lens and a CCD (charge coupled device). The Linnik microscope has two arms, which are referred to as a reference arm and a measurement arm. The reference mirror is placed on the focal plane of the objective lens in the reference arm, while a tool face to be measured is placed on the focal plane of the other objective lens in the measurement arm. Interference fringes are generated in accordance with the optical path difference (OPD) between the reference arm and the measurement arm.

Compared with the Mirau configuration having a reference mirror in an objective lens in its measurement arm, the optical setup for the Linnik configuration tends to become more complicated. Alignments of each optical component in the Linnik configuration for better quality fringe patterns are also time-consuming tasks. However, the Linnik configuration is compatible with the micro-optical probe described in the previous subsection, since it does not have a reference mirror in the optical path of the micro-optical probe in the measurement arm. Furthermore, the Linnik configuration can employ objective lenses with a higher numerical aperture (N. A.), which has advantage of wide-range measurement of round-shaped surfaces [24].

\subsection{Proposed optical setup for multi-dimensional measurement of a diamond cutting tool}

Figure 2(c) shows a concept of the proposed method for multi-dimensional measurement of a diamond cutting tool. The micro-optical probe component and the Linnik microscope component are combined with each other in such a way that the components share the same measuring optical 
axis. In the case of the interferometric microscope employing an incoherent light such as white light as a light source, fringe patterns with maximum contrast can be acquired when a tool face is placed at the focal plane of the objective lens. Meanwhile, the diameter of the micro-optical probe for tool edge contour measurement is minimized on the focal plane. Therefore, by using the information of the fringe patterns, the tool edge can be precisely placed on the focal plane of the objective lens. Furthermore, tilt angles of a measuring tool face can also be assured by using the fringe patterns captured by the CCD in the Linnik microscope component. Measurement condition of the micro-optical probe can thus be optimized by the combination with the Linnik microscope component.

Figure 3 shows an example of a sequence for multi-dimensional measurement of a diamond cutting tool. At first, by using a function of the Linnik microscope component, a cutting tool will be positioned at the focal plane of the objective lens in the measurement arm. Tilt angles of the tool face with respect to the micro-optical probe will also be adjusted at this stage. Secondly, the $Y$-directional position of the tool edge will be adjusted precisely with respect to the micro-optical probe by monitoring the PD outputs in the micro-optical probe component. After that, a scanning path of the micro-optical probe will be generated by using a two-dimensional image of the tool face acquired by the Linnik microscope component. A tool edge will then be scanned by the micro-optical probe to acquire the tool edge contour. By employing the images acquired by the Linnik microscope component, tool face measurement can be carried out in a large range with an employment of stitching process. Finally, the tool flank face will be measured by rotating the cutting tool.

\section{Design of the optical configuration}

To verify the feasibility of the proposed concept for multi-dimensional measurement of a diamond cutting tool, a prototype of the measuring instrument is designed and constructed. Figure 4 shows the designed optical configuration for the measuring instrument. In the design, the 
micro-optical probe component and the Linnik microscope component are combined with each other. The optical axes of the micro-optical component and the measurement arm in the Linnik microscope component are aligned to be consistent with each other by using a polarized beam splitter (PBS) and a beam splitter (BS).

As a light source for the micro-optical probe component, a LD with a wavelength $\lambda_{\text {laser }}$ of 405 $\mathrm{nm}$ is employed. Regarding the principle of the proposed micro-laser probe for tool edge contour measurement, intensity deviation of the laser beam affects the measurement accuracy. In the prototype design, a pair of photodiodes $\mathrm{PD}_{\mathrm{R}}$ and $\mathrm{PD}_{\mathrm{M}}$ are then employed to eliminate the influence of the intensity deviation of the laser beam. PDM is used to monitor the intensity of the laser beam passed through the focal plane of the objective lens in the measurement arm, in which a cutting tool to be measured is inserted. Meanwhile, $\mathrm{PD}_{\mathrm{R}}$ is employed to monitor the intensity deviation of the laser beam emitted from the LD. A linearly-polarized laser beam emitted from the LD is divided by using the PBS. The angle of the polarization plane of the laser beam with respect to the PBS is adjusted in such a way that a part of the laser beam can transmit the PBS and reach the PDR. Stability of the laser oscillation is also important for highly-stable measurement. $\lambda / 4$ plates are therefore employed in the optical setup so that an optical isolator can be arranged. The ideal diameter of the laser beam $d_{0}$ on the focal plane of the objective lens in the measurement arm can be calculated as follows [25]:

$$
d_{0}=M^{2} \frac{f \lambda_{\text {laser }}}{D}
$$

where $M^{2}$ is a quality factor referred to as the M-squared factor, $f$ is a focal length of the objective lens and $D$ is a incident beam diameter. In the design, objective lenses with a focal length $f$ of 10 $\mathrm{mm}$, a focal depth of $1.6 \mu \mathrm{m}$ and numerical aperture (NA) of 0.42 are employed. A diameter of the collimated laser beam, which corresponds to $D$, is adjusted to be approximately $6 \mathrm{~mm}$. Therefore, if we assume $M^{2}$ to be 2 , the spot diameter $d$ is calculated to be approximately $1.35 \mu \mathrm{m}$.

As a light source for the Linnik microscope component, a light emitting diode (LED) is employed. A collector lens and a field lens are employed to set up Köhler illumination [23]. The 
LED with a spectrum ranging from $420 \mathrm{~nm}$ to $800 \mathrm{~nm}$ is chosen to avoid unnecessary interference with the micro-laser probe. Regarding the spectrum of the light source [26], interference signal has been simulated. The interference signal $I$, which is a function of the Z-directional displacement $\Delta z$ with respect to the focal plane of the objective lens, can be calculated as a summation of interference signals $I_{\mathrm{c}}(\Delta z, \lambda)$ over a range of waveform $\lambda$, while including characteristics of the light spectrum [23]:

$$
I(\Delta z)=\int I_{c}(\Delta z, K(\lambda)) V(\lambda) \varpi(\lambda) d \lambda
$$

where $V(\lambda)$ and $\varpi(\lambda)$ are the spectrum of the light source and spectrum characteristics of optics used in the interferometric microscope. $K(\lambda)(=2 \pi / \lambda)$ is the angular wave number for a spectral contribution. Figure 5(a) shows the calculated interference signal. $V(\lambda)$ and $\varpi(\lambda)$ shown in the specification sheet of each optical component are employed in the calculation. A period of the interference signal around the focal plane of the objective lens is found to be approximately $280 \mathrm{~nm}$. Figure 5(b) shows a deviation of the spot diameter $d$ of the micro-optical probe, which can be calculated by the following equation [27]:

$$
d=d_{0} \sqrt{1+\left(\frac{\lambda_{\text {laser }} \Delta z}{\pi D^{2}}\right)^{2}}
$$

These results indicate that the deviation of the spot diameter of the micro-optical probe can be suppressed to be within $0.01 \mu \mathrm{m}$ when the tool cutting edge is positioned within the range of \pm 140 $\mathrm{nm}$ from the focal plane, which corresponds the period of the interference signal. Therefore, the Linnik microscope component is effective not only in measuring tool faces but also in optimizing the micro-optical probe component.

A photograph of the developed prototype is shown in Fig. 6. An optical cage system was employed since it was developed as the prototype for verifying the feasibility of the proposed method. For on-machine measurement, the measuring instrument is required to be designed in a compact size, which will be carried out as future work. Figure 7 shows the block diagram of the 
developed measuring instrument. In the setup, the cutting tool is mounted on a precision $X Y Z$-stage system composed of a PZT Z-stage and a pair of one-axis servo motor stages. The in-plane motion is given to a cutting tool by the pair of one-axis servo motor stages for tool edge contour measurement with the micro-optical probe. The Z-directional motion for measurement of the Linnik microscope component and for the fine adjustment of the micro-optical probe is generated by the PZT Z-stage. The XYZ-coordinates of the tool are monitored by the displacement sensors in the XYZ-stage system, and are captured by a personal computer via the RS-232C interface. The photocurrent signals from the photodiodes $\left(\mathrm{PD}_{\mathrm{M}}\right.$ and $\left.\mathrm{PD}_{\mathrm{R}}\right)$, the amounts of which correspond to the intensity of the captured light, are converted into voltage output signals by using current-voltage converting circuits, and are monitored by an oscilloscope. As the image sensor for the Linnik microscope component, the CCD with a pixel number of $1296 \times 966$ and a pixel size of $3.75 \mu \mathrm{m} \times$ $3.75 \mu \mathrm{m}$ is employed. A scanning motion of the PZT Z-stage and the image capturing of the CCD are synchronized by using a periodic signal generated from a function generator.

\section{Experiments}

By using the developed measuring instrument, feasibility of the proposed method was verified. Since the developed measuring instrument was a prototype, in this paper, only basic performances of the micro-laser probe in the multi-dimensional measuring station were evaluated. At first, the spot size of the micro-optical probe on the focal plane of the objective lens was evaluated. In the evaluation, a knife edge method [28] was employed since commercial instruments such as a beam profiler could not measure a micro-sized beam spot. A knife edge was mounted on the $X Y Z$-stage system and was scanned in the $X Y$-directions, while output signal from the $\mathrm{PD}_{\mathrm{M}}$ was being monitored. Figure 8 shows the relationship between the $\mathrm{PD}_{\mathrm{M}}$ output and the displacement of the knife edge. The diameter of the micro-optical probe was evaluated to be $1.8 \mu \mathrm{m}$ (FWHM). This value was larger than expected regarding the results in the previous section, the reasons of which were possibly due to the collimating lens employed in the optical setup or its misalignment. 
Although further careful alignment of each optical component had a possibility of reducing the spot size, the spot diameter was enough small to be used as the micro-optical probe.

Following the verification test for the spot diameter, sensitivity of the micro-laser probe for tool edge contour measurement was evaluated in experiments. In the test, the $X Z$-positions of the micro-optical probe were held stationary, while the $Y$-directional motion was given to the cutting tool, whose rake face was aligned to be consistent with the focal plane of the objective lens. Figure 9 shows the relationship between the $Y$-directional tool displacement and the laser probe output. In the $Y$-directional range of $\pm 250 \mathrm{~nm}$, the sensitivity of the micro-optical probe was evaluated to be approximately $6.744 \mathrm{~nm} / \%$. According to the noise level of the micro-optical probe $(0.03 \%$ of the normalized PD output), it was verified that the laser probe had a resolution of better than $1 \mathrm{~nm}$ for tool edge contour measurement.

By using the same setup, the influence of the deviation of the $Z$-position of the micro-optical probe on tool edge contour measurement was evaluated. In the experiment, at first, the $X Y$-positions of the micro-optical probe were adjusted in such a way that a half of the micro-optical probe was blocked by the tool rake face. The micro-optical probe was then held stationary in the $X Y$-directions, while the Z-directional motion was applied to the cutting tool. During the experiment, deviation of the output signal from the $\mathrm{PD}_{\mathrm{M}}$ was monitored. Figure 10 shows a deviation of the $\mathrm{PD}_{\mathrm{M}}$ output caused by the $Z$-directional displacement of the tool edge with respect to the micro-optical probe. In the figure, deviation of the $\mathrm{PD}$ M output was converted to the corresponding error for tool edge contour measurement. This result shows that large measurement error can occur with the $Z$-directional tool displacement even if the tool cutting edge is placed in the range of the focal depth of the objective lens $( \pm 1.6 \mu \mathrm{m})$. A tool edge is therefore desired to be positioned at the focal plane of the objective lens as precise as possible during the scanning of the laser spot for precise measurement of the tool edge contour.

An influence of the white-light illumination for the Linnik microscope component on the micro-optical probe component was also verified in experiments. Figure 11 shows voltage output 
waveform of the $\mathrm{PD}_{\mathrm{M}}$ in the micro-optical probe before and after the switching of the LED illumination for the Linnik microscope component. It was verified that the micro-optical probe was not be affected by the existence of the LED illumination.

Experiments were also carried out to verify the basic performances of the Linnik microscope component. In the tests, interference signals were acquired by using both a flat mirror surface and a rake face of a diamond cutting tool as measuring targets. Figure 12 shows the acquired interference signals detected at a certain cell on the CCD used in the Linnik microscope component. Figure 12(a) and (b) shows the interference signals observed by measuring the flat mirror surface and the tool rake face, respectively. The periods of measured interference signals around the focal plane of the objective lens were found to be approximately $300 \mathrm{~nm}$, which were slightly larger than that acquired in the simulation $(280 \mathrm{~nm})$ described in the previous section. In addition, a coherence length observed in the experiment was slightly longer than that shown in the simulation results. In the optical path of the Linnik microscope, two filters were employed; one was placed beneath the measuring target, while the other was placed in front of the imaging lens and the CCD. Filters in the system made the spectrum bandwidth narrow, resulting in the larger period of the measured interference signals and the longer coherence length. Compared with the result for the flat mirror surface, visibility of the interference signal was found to be degraded in the measurement result of the tool rake face; which was considered to be due to a low reflectivity of the diamond surface. However, from these results, it was verified that the developed Linnik microscope component could measure surfaces with a low reflectivity such as a diamond surface.

Measurements of tool faces were then carried out by using the developed instrument. A cutting tool with a round nose with a nose radius of $2 \mathrm{~mm}$, which had been used for diamond turning process, was employed as a measuring target. Figure 13(a) and (b) show the two-dimensional image of the CCD and the tool rake face profile measured by the developed instruments, respectively. In the measured profile, slight wears with depth of approximately $300 \mathrm{~nm}$ generated during the diamond turning process were clearly observed on the rake face. It should be noted that a coarse 
tool edge contour can be extracted from results acquired in the Linnik microscope component, and can be utilized to generate a scanning path of the micro-optical probe for fine tool edge contour measurement. Figure 14 shows its example; the out-of-roundness of the worn tool edge contour extracted from the image shown in Fig. 13(b). Fine measurement of tool edge contours with arbitrary shape can be carried out by the micro-optical probe component with the information of the extracted coarse tool edge contour. A tool flank face, a schematic of which is shown in Fig. 15(a), was also evaluated by the developed instrument. Figure 15(b) shows a measured profile of the flank face of a diamond cutting tool with a nose radius of $1 \mathrm{~mm}$. A tool clearance face was also successfully measured by the developed instrument. It should be noted that we have focused on the design and basic tests of the combined optical system for multi-dimensional measurement of a diamond cutting tool in this paper. To further verify the feasibility of the proposed method, a fair comparison with other methods will be carried out in future work by using the further optimized optical setup. Verification of the effects of the Linnik microscope component on the optimization of the micro-optical probe component for tool edge contour measurement, and on-machine multi-dimensional measurement of a diamond cutting tool will also be carried out as future work.

\section{Conclusions}

An optical measuring instrument for evaluation of tool edge contour and tool faces was proposed, and its design study was carried out. An optical configuration consisting of a micro-optical probe component and a Linnik microscope component was designed in such a way that the optical axes of both the components were consistent with each other. A prototype of the measuring instrument was developed by using an optical cage system, and its feasibility was tested in experiments. It was verified that the micro-optical probe had a diameter of less than $2 \mu \mathrm{m}$ on the focal plane of the objective lens, and achieved resolution of better than $1 \mathrm{~nm}$ for tool edge contour measurement. It was also verified that there was not any significant crosstalk between the micro-optical probe component and the Linnik microscope component. Furthermore, basic 
performances of the developed Linnik microscope component were also evaluated by experiments. A rake face of the diamond cutting tool with a nose radius of $2 \mathrm{~mm}$ was successfully measured with a vertical resolution of better than $1 \mathrm{~nm}$. In addition, a tool flank face with a round surface of a cutting tool with a nose radius of $1 \mathrm{~mm}$ was successfully measured by the developed system. Further detailed verification tests and on-machine multi-dimensional measurement of a diamond cutting tool will be carried out as future work.

\section{Acknowledgements}

A part of this project was supported by Japan Society for the Promotion and Science (JSPS) and Japan Science and Technology Agency (JST) (A-STEP, 241FT0173).

\section{References}

[1] T. Moriwaki, K. Okuda, Machinability of Copper in Ultra-Precision Micro Diamond Cutting, CIRP Annals-Manufacturing technology, 38 (1989) 115-118.

[2] F. Z. Fang and V. C. Venkatesh, Diamond cutting of silicon with nanometric finish, CIRP Annals-Manufacturing technology, 47 (1998) 45-49.

[3] W. J. Zong, Z. Q. Li, T. Sun, K. Cheng, D. Li and S. Dong, The basic issues in design and fabrication of diamond-cutting tools for ultra-precision and nanometric machining, Int. J. of Machine Tools \& Manufacture, 50 (2009) 411-419.

[4] A. A. G. Bruzzone, H. L. Costa, P. M. Lonardo, D. A. Lucca, Advances in engineered surface for functional performance, CIRP Annals-Manufacturing technology, 57 (2008) 750-769.

[5] F. J. Chen, S. H. Yin, H. Huang, H. Ohmori, Y. Wang, Y. F. Fan, Y. J. Zhu, Profile error compensation in ultra-precision of aspheric surfaces with on-machine measurement, Int. J. of Mach. Tools \& Manuf., 50 (2010) 480-486.

[6] N. Kawasegi, H. Sugimori, H. Morimoto, N. Morita and I. Hori, Development of cutting tools with microscale and nanoscale textures to improve frictional behavior, Precision Engineering, 33 (2009) 248-254.

[7] D. Kono, A. Matsubara, I. Yamaji and T. Fujita, High-precision machining by measurement 
and compensation of motion error, Int. J. of Machine Tools \& Manufacture, 48 (2008) 1103-1110.

[8] D. K. Born and W. A. Goodman, An empirical survey on the influence of machining parameters on tool wear in diamond turning of large single-crystal silicon optics, Precision Engineering, 25 (2001) 247-257.

[9] S. Asai, Y. Taguchi, K. Horio, T. Kasai, A. Kobayashi, Measuring the very small cutting-edge radius for a diamond tool using a new kind of SEM having two detectors, CIRP Annals-Manufacturing technology, 39 (1990) 85-88.

[10] J. Drescher, Scanning electron microscopic technique for imaging a diamond tool edge, Precision Engineering, 15 (1993) 112-114.

[11] A. Weckenmann and K. Nalbantic, Precision measurement of cutting tools with two matched optical 3D-sensors, CIRP Annals-Manufacturing technology, 52 (2003) 443-446.

[12] H. H. Shahabi and M. M. Ratnam, On-line monitoring of tool wear in turning operation in the presence of tool misalignment, Int. J. of Advanced Manufacturing Technology, 38 (2007) 718-727.

[13] T. Y. Lim, M. M. Ratnam, Edge detection and measurement of nose radii of cutting tool inserts from scanned 2-D images, Optics and Lasers in Engineering, 50 (2012) 1628-1642.

[14] Y. Wang, N. Suzuki, E. Shamoto and Q. Zhao, Investigation of tool wear suppression in ultraprecision diamond machining of die steel, Precision Engineering, 35 (2011) 677-685.

[15] W. Grzesik and K. Zak, Friction quantification in the oblique cutting with CBN chafered tools, Wear, 304 (2013) 36-42.

[16] http://www.alicona.com/home/products/if-edgemaster.html (accessed 28 January 2015).

[17] http://www.mitakakohki.co.jp/industry/nh_series/lineup/nh-3sp.html (accessed 28 January 2015).

[18] D. A. Lucca and Y. W. Seo, Effect of tool edge geometry on energy dissipation in ultraprecision machining, CIRP Annals-Manufacturing technology, 42 (1993) 83-86. 
[19] W. Gao, T. Motoki, S. Kiyono, Nanometer edge profile measurement of diamond cutting tools by atomic force microscope with optical alignment sensor, Precision Engineering, 30 (2006) 396-405.

[20] W. Gao, T. Asai, and Y. Arai, Precision and fast measurement of 3D cutting edge profiles of single point diamond micro-tools, CIRP Annals-Manufacturing technology, 58 (2009) 451-454.

[21] S. H. Jang, Y. Shimizu, S. Ito and W. Gao, A micro optical probe for edge contour evaluation of diamond cutting tool, J. Sens. Sens. Syst., 3 (2014) 69-76.

[22] S. H. Jang, Y. Shimizu, S. Ito and W. Gao, Development of an optical probe for evaluation of tool edge geometry, Journal of Advanced Mechanical Design, Systems, and Manufacturing, 8 (2014) No.14-00104 (10pp).

[23] R. K. Leach, Optical Measurement of Surface Topography, Springer, London (2011).

[24] J. W. Chesna, Y. Shimizu, R. K. Leach, C. L. Giusca and A. J. Henning, On the use of mercury sessile drops as reference artefacts for the calibration of optical surface topography measuring instruments, Proceedings of ASPE, St Paul, USA (2013) 74-77.

[25] T. F. Johnston, Jr., Beam propagation $\left(\mathrm{M}^{2}\right)$ measurement made as easy as it gets: the four-cuts method, Applied Optics, 37 (1998) 4840-4850.

[26] http://www.thorlabs.co.jp/ (Accessed 28 January 2015).

[27] E. Hecht, Optics (4th Ed.), Addison Wesley, CA, USA (2002).

[28] J. M. Khosrofian and B. A. Garetz, Measurement of a Gaussian laser beam diameter through the direct inversion of knife-edge data, Applied Optics, 22 (1983) 3406-3410. 


\section{Figure captions}

Fig. 1 A schematic of a single-point diamond cutting tool

Fig. 2 A schematic of the proposed measuring instrument

(a) Micro-optical probe for tool edge contour measurement

(b) Linnik microscope for tool face measurement

(c) A proposed optical setup for multi-dimensional measurement of a diamond cutting tool

Fig. 3 An example of the measurement procedure

Fig. 4 Optical configuration of the developed instrument

Fig. 5 Simulation on the interference signal and the spot diameter of the micro-optical probe

(a) Interference signal

(b) Spot diameter of the micro-optical probe

Fig. 6 Photograph of the developed prototype

Fig. 7 Block diagram of the developed measuring station

Fig. 8 Measured spot size

Fig. 9 Sensitivity of the micro-optical probe

Fig. 10 Measurement error due to the positioning error of the tool edge in the $Z$-direction

Fig. 11 Variation of the voltage output waveform from the photodiode $\mathrm{PDM}_{\mathrm{M}}$ at the switching of the LED illumination

Fig. 12 Measured interference signals

(a) Interference signal measured on the flat mirror surface

(b) Interference signal measured on the diamond tool face

Fig. 13 Measured tool rake face

(a) CCD image

(b) Profile measured by the Linnik microscope component

Fig. 14 Tool edge contour

Fig. 15 Tool flank face measured by the developed instrument

(a) A schematic of a flank face

(b) Measured flank face 


\section{Designed optical setup}

$$
\stackrel{Z}{\longrightarrow} X
$$

Micro-optical probe (for edge contour measurement)

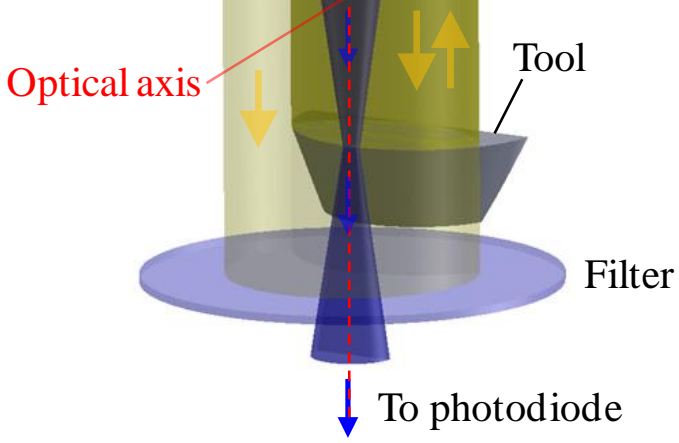

Tool edge contour (coarse measurement)

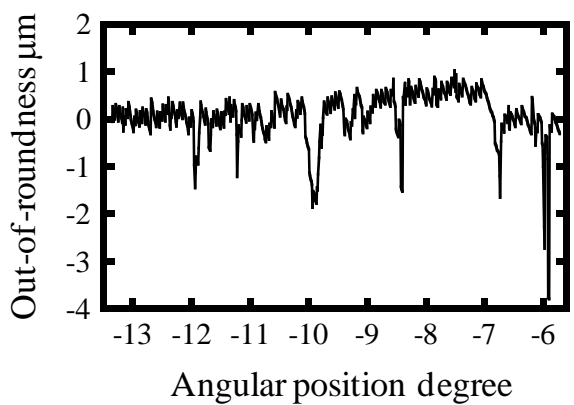

(for tool face measurement)

Filter
Tool rake face

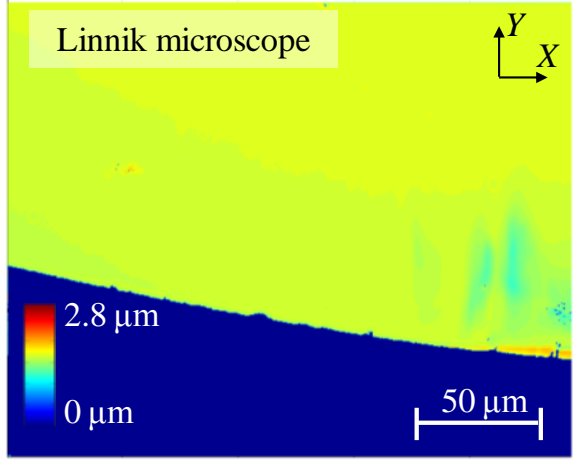

$\underline{\text { Tool flank face }}$

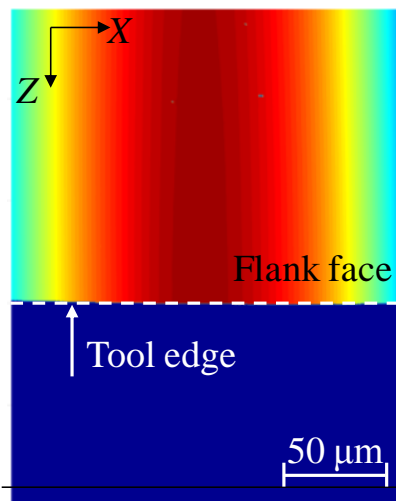

Height $(Y)$

$0 \mu \mathrm{m}$

$-7 \mu \mathrm{m}$ 


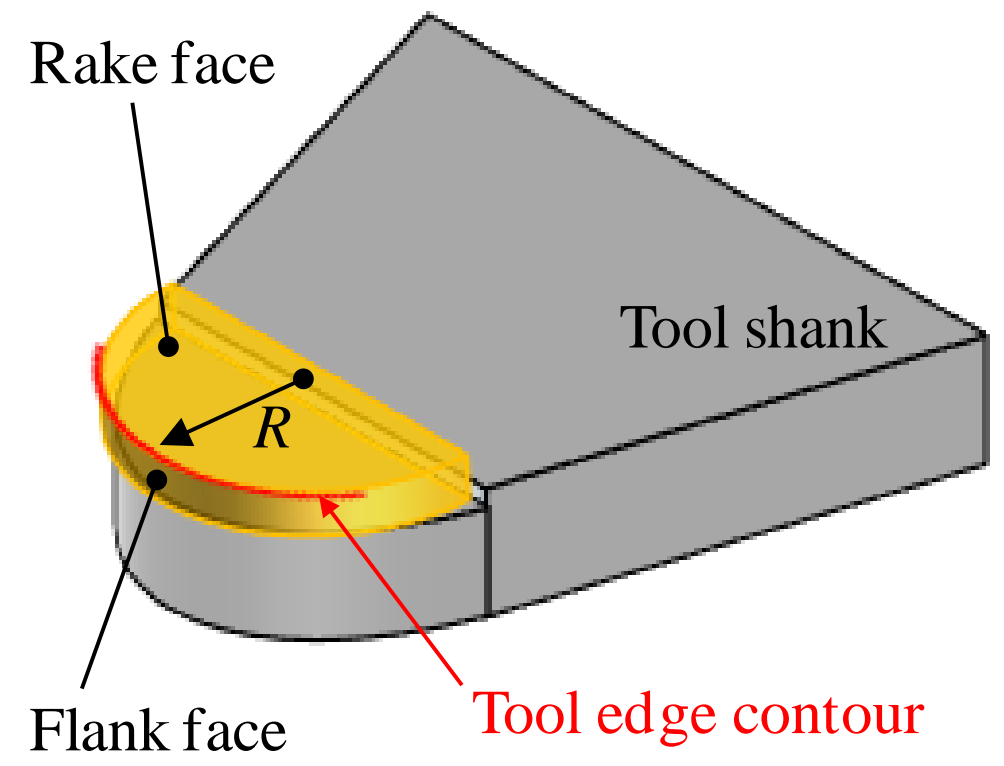

Fig. 1 A schematic of a single-point diamond cutting tool 


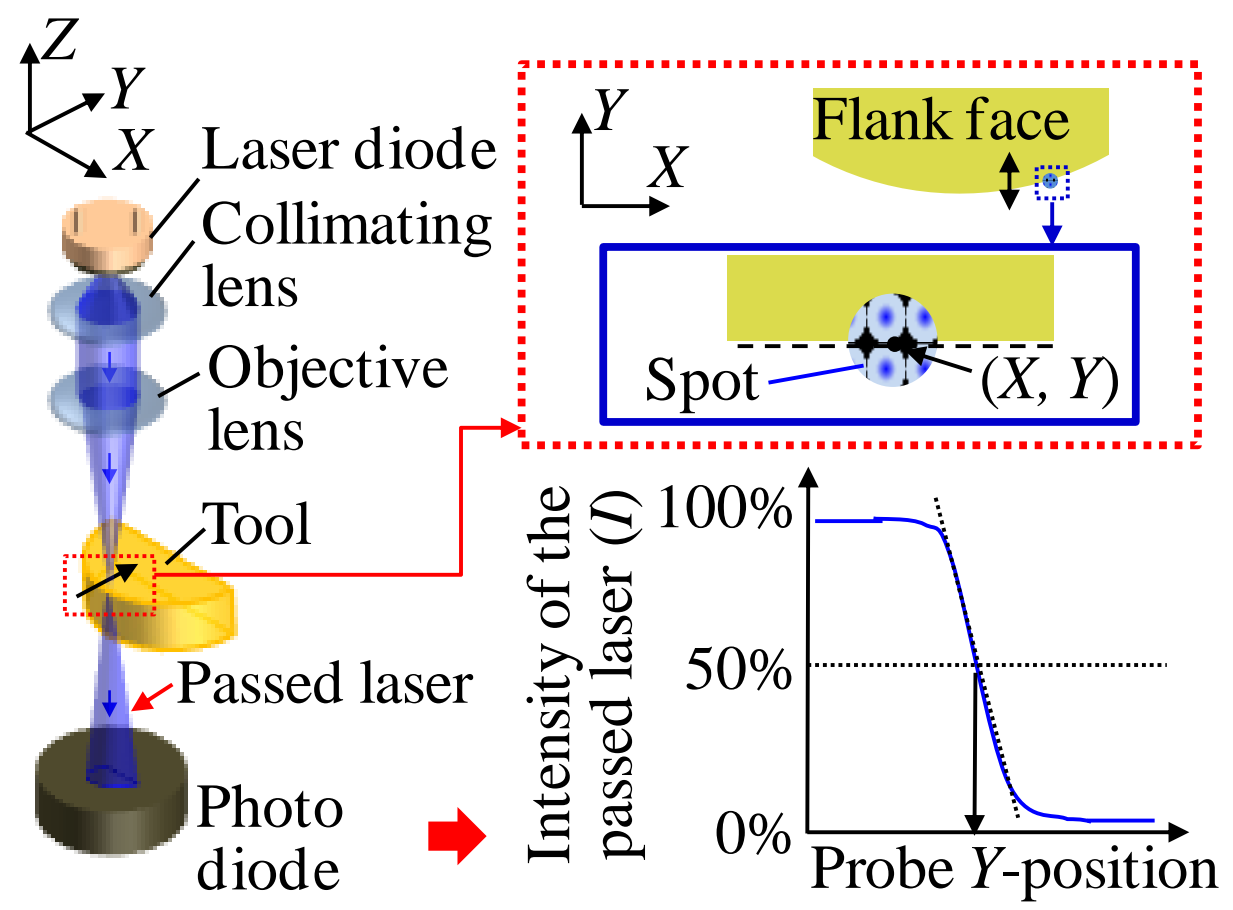

(a) Micro-optical probe for tool edge contour measurement

Fig. 2 A schematic of the proposed measuring instrument 


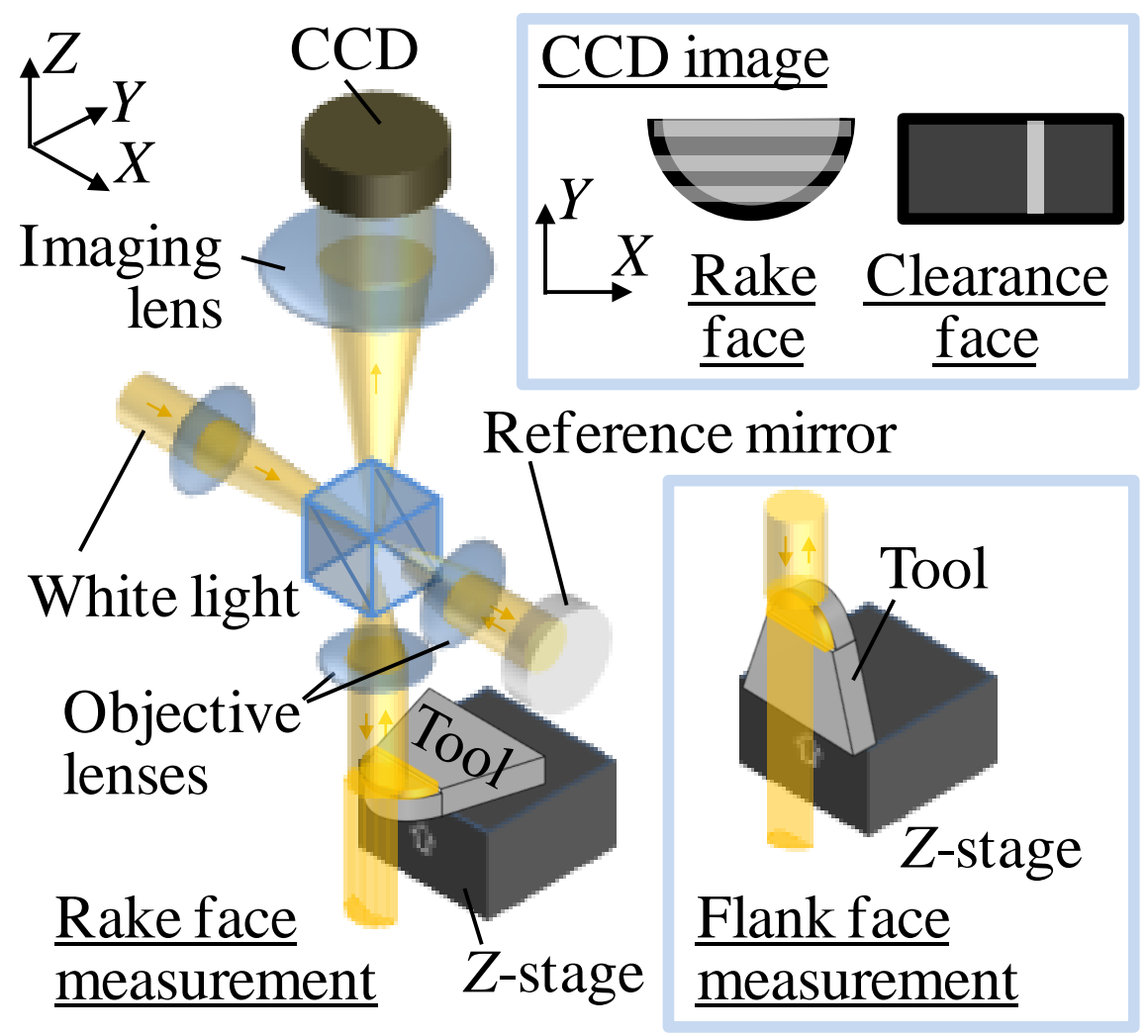

(b) Linnik microscope for tool face measurement

Fig. 2 A schematic of the proposed measuring instrument 


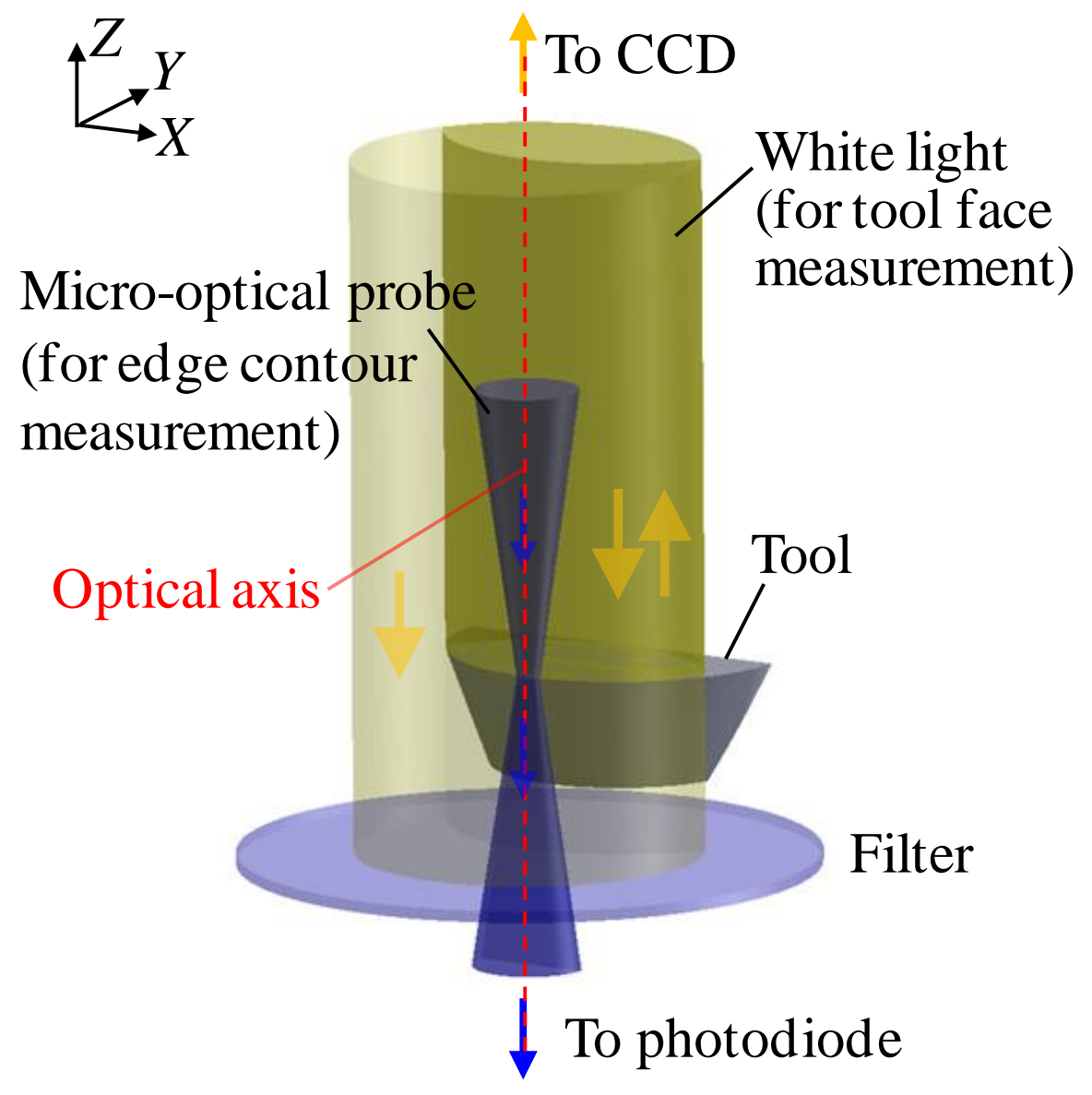

(c) A proposed optical setup for multi-dimensional measurement of a diamond cutting tool

Fig. 2 A schematic of the proposed measuring instrument 


\section{Start}

Coarse positioning of the tool edge with respect to the micro-optical probe

Fine a lignment for tool face mea surement

(Tilts and focus)

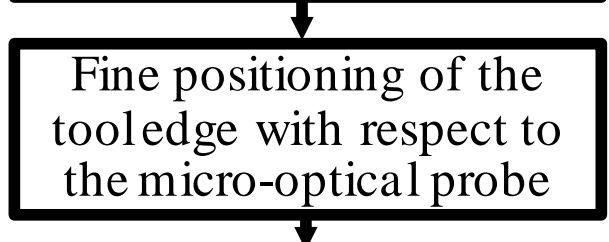

Toolrake face measurement by the Linnik microscope

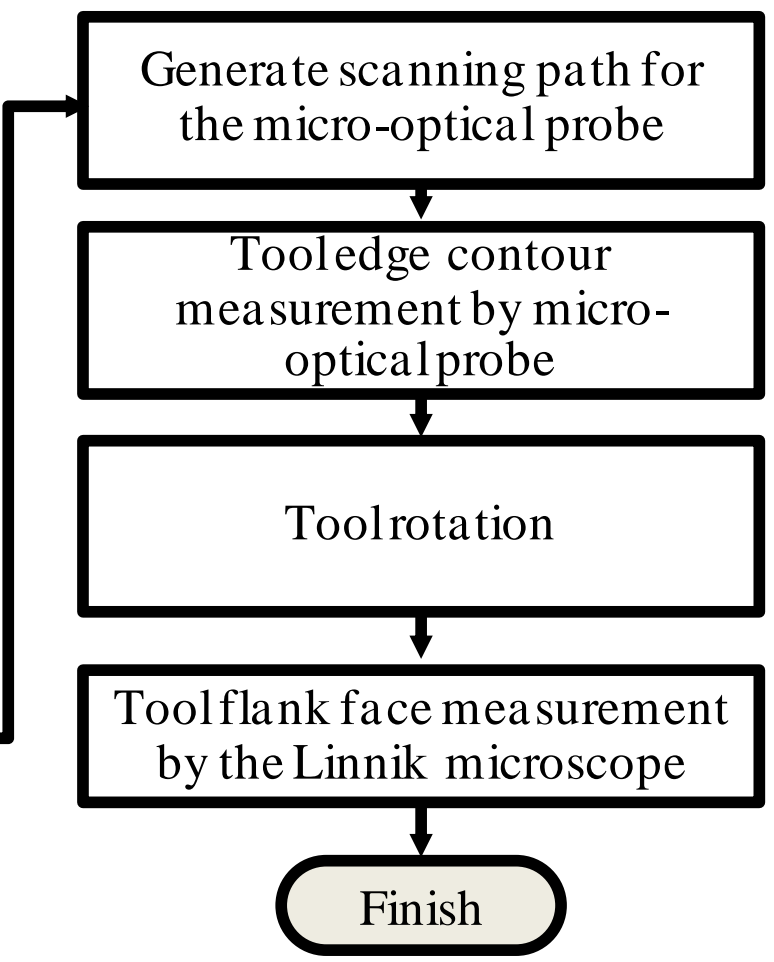

Fig. 3 An example of the measurement procedure 


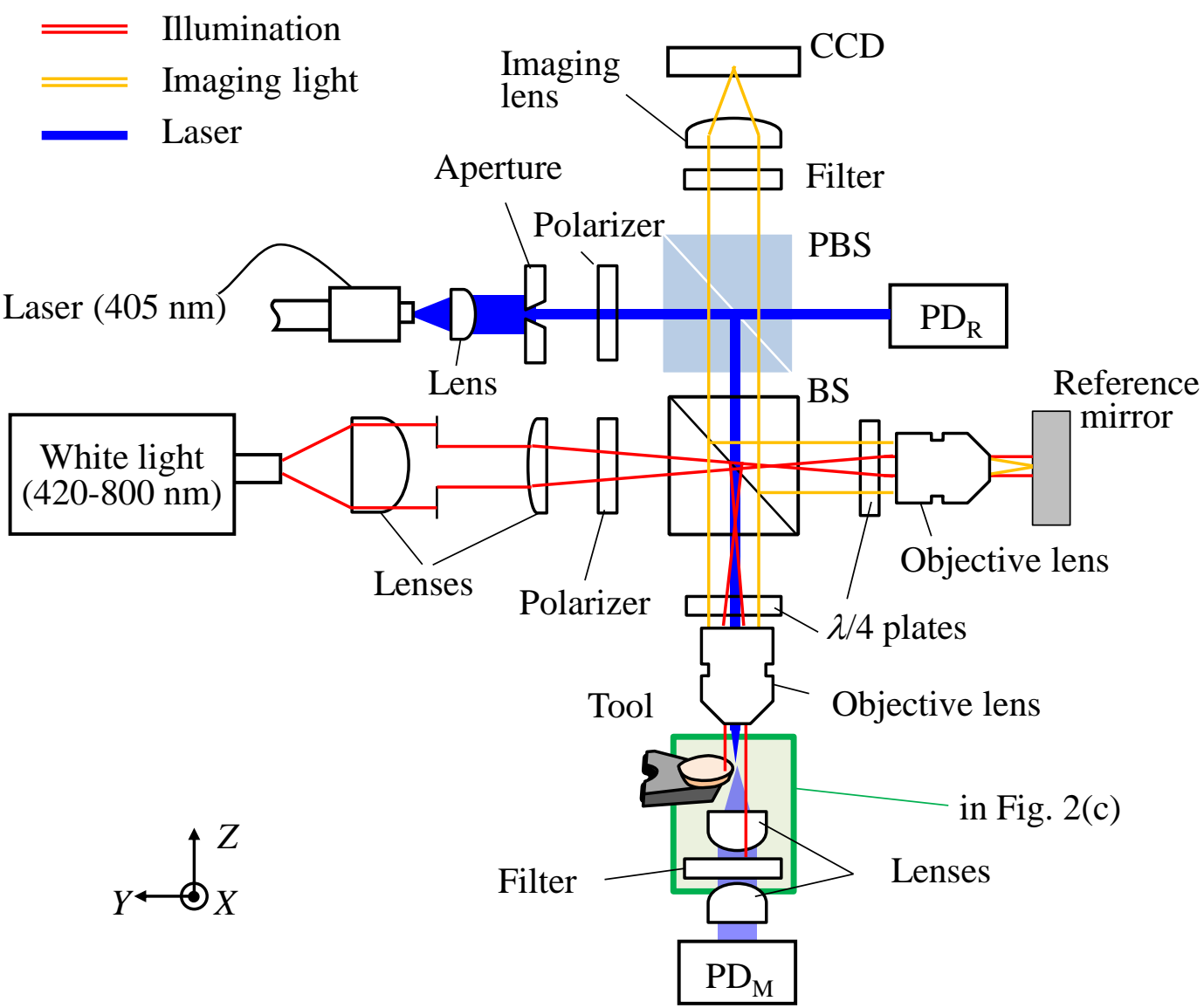

Fig. 4 Optical configuration of the developed instrument 


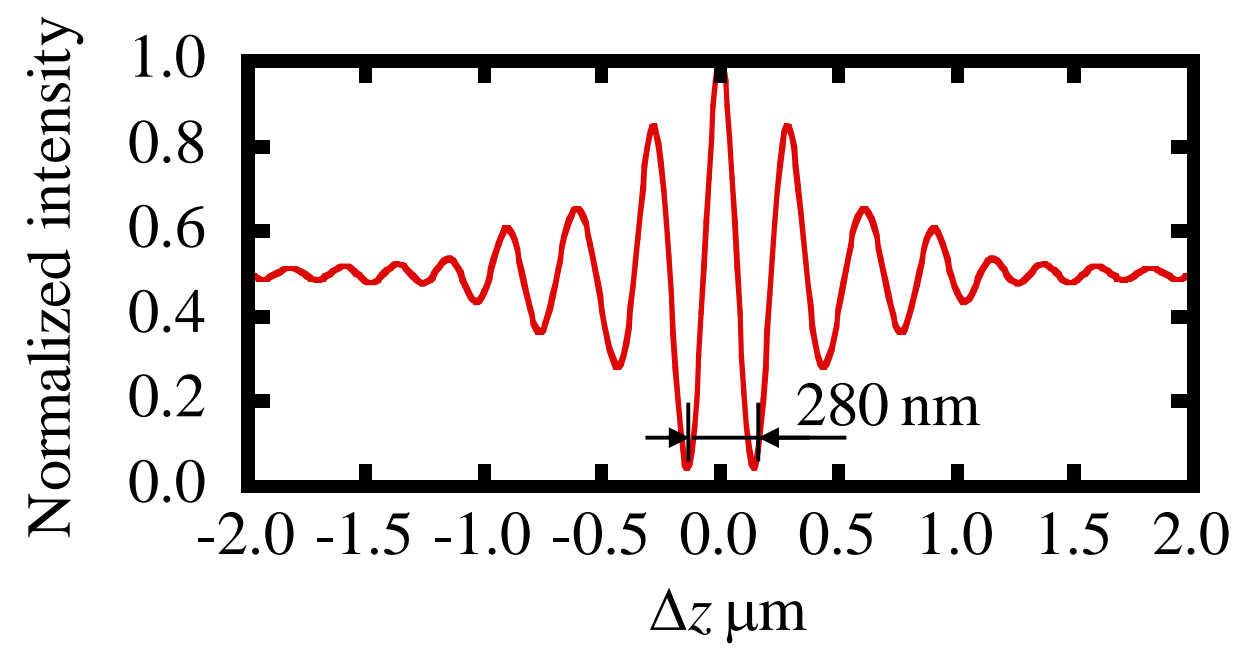

(a) Interference signal

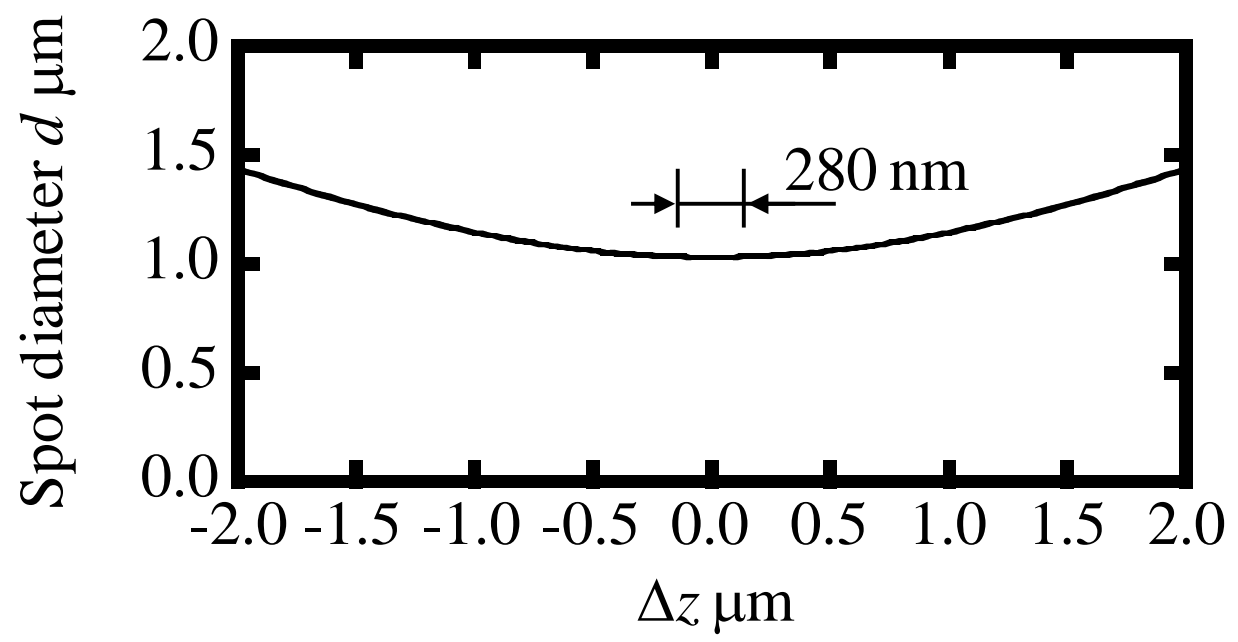

(b) Spot diameter of the micro-optical probe

Fig. 5 Simulation on the interference signal and the spot diameter of the micro-optical probe 


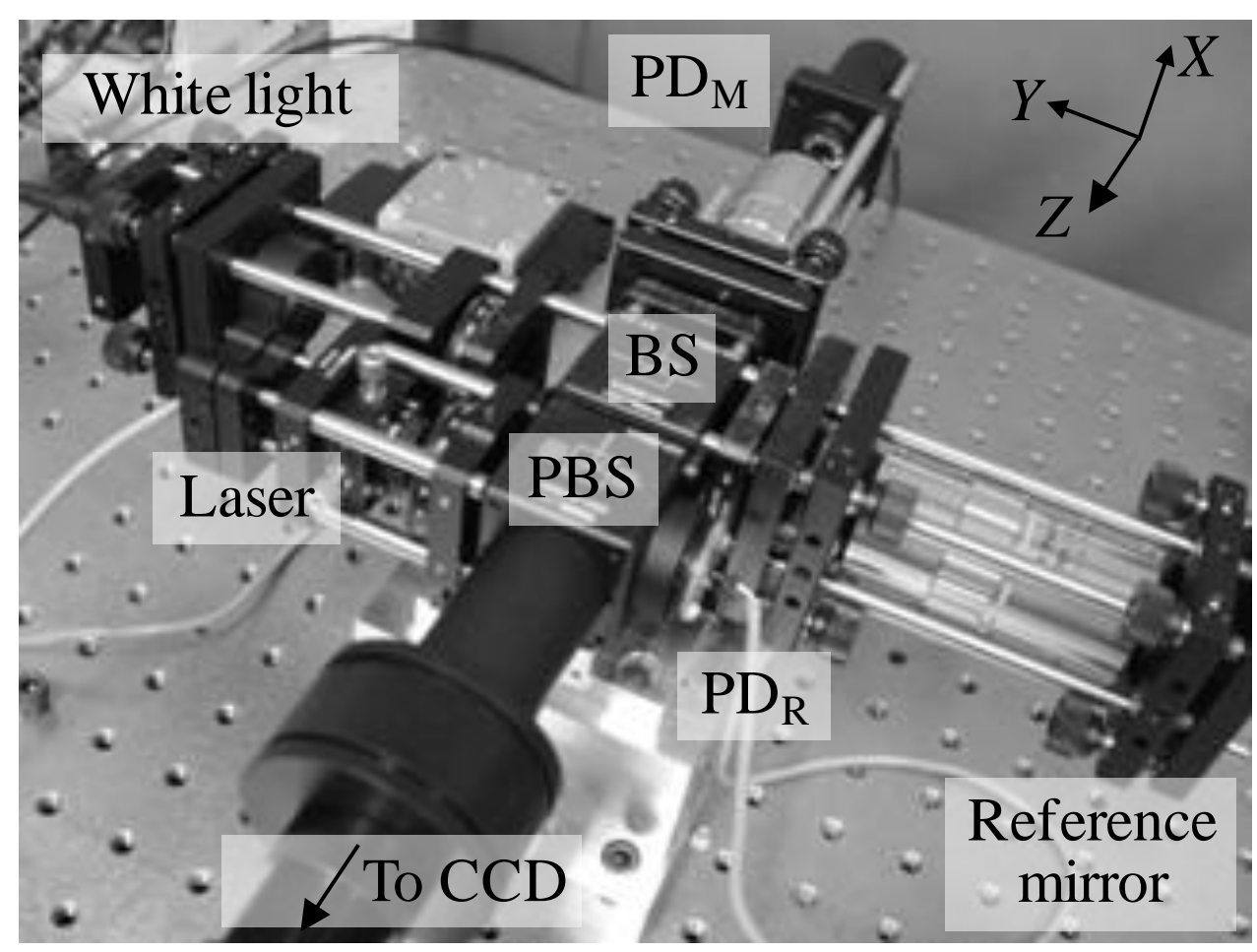

Fig. 6 Photograph of the developed prototype 


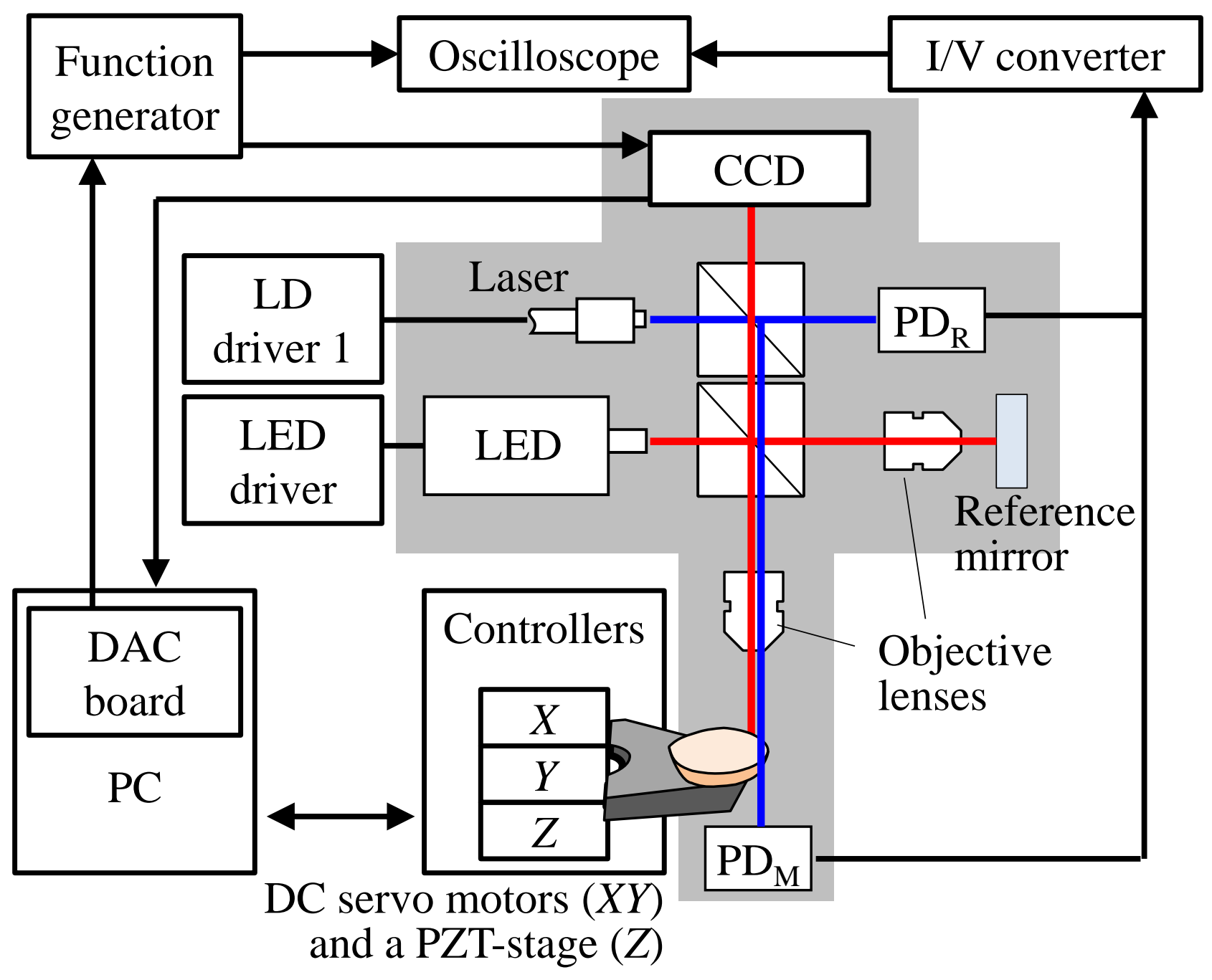

Fig. 7 Block diagram of the developed measuring station 


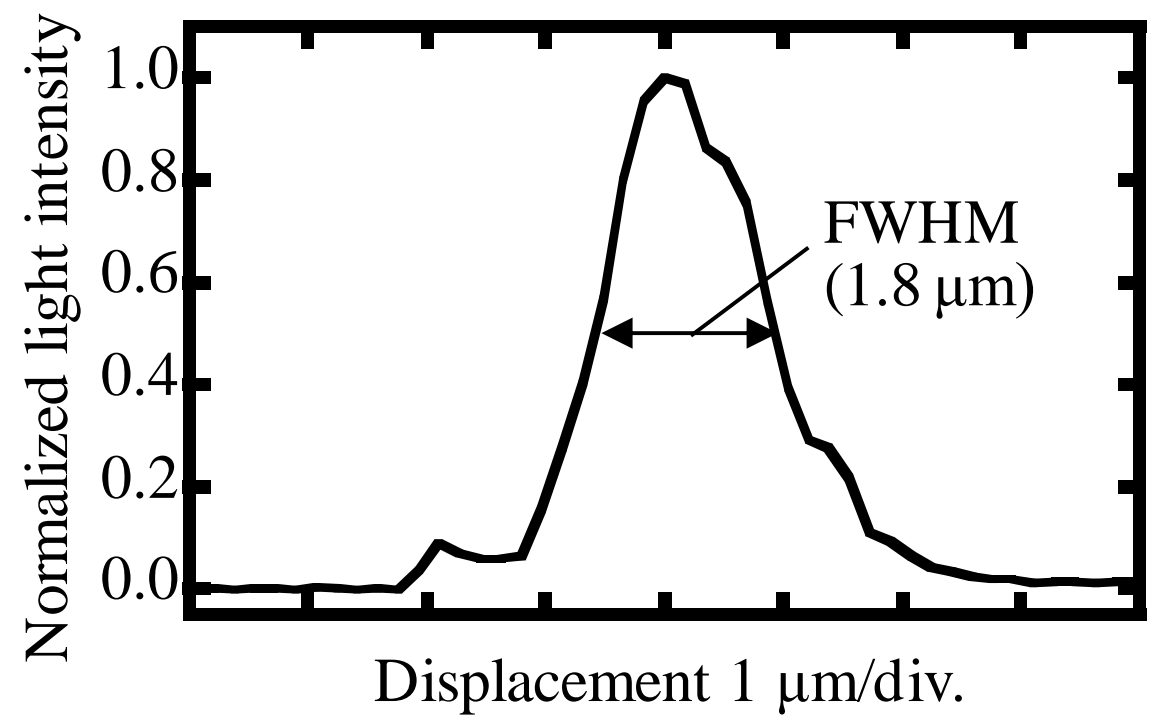

Fig. 8 Measured spot size 


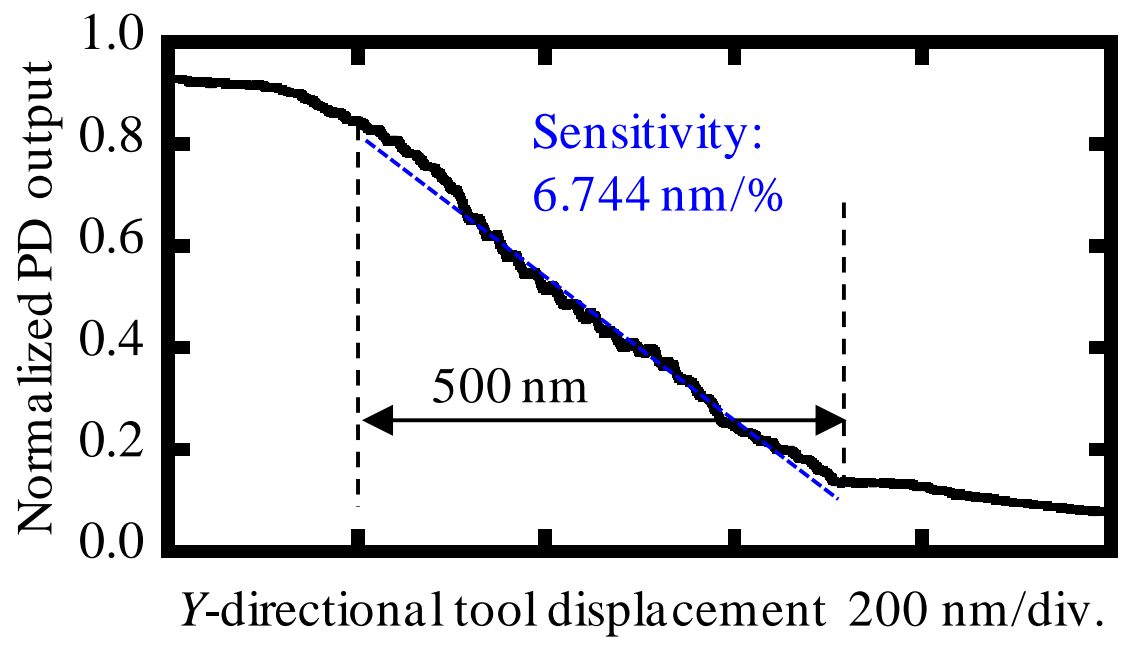

Fig. 9 Sensitivity of the micro-optical probe 


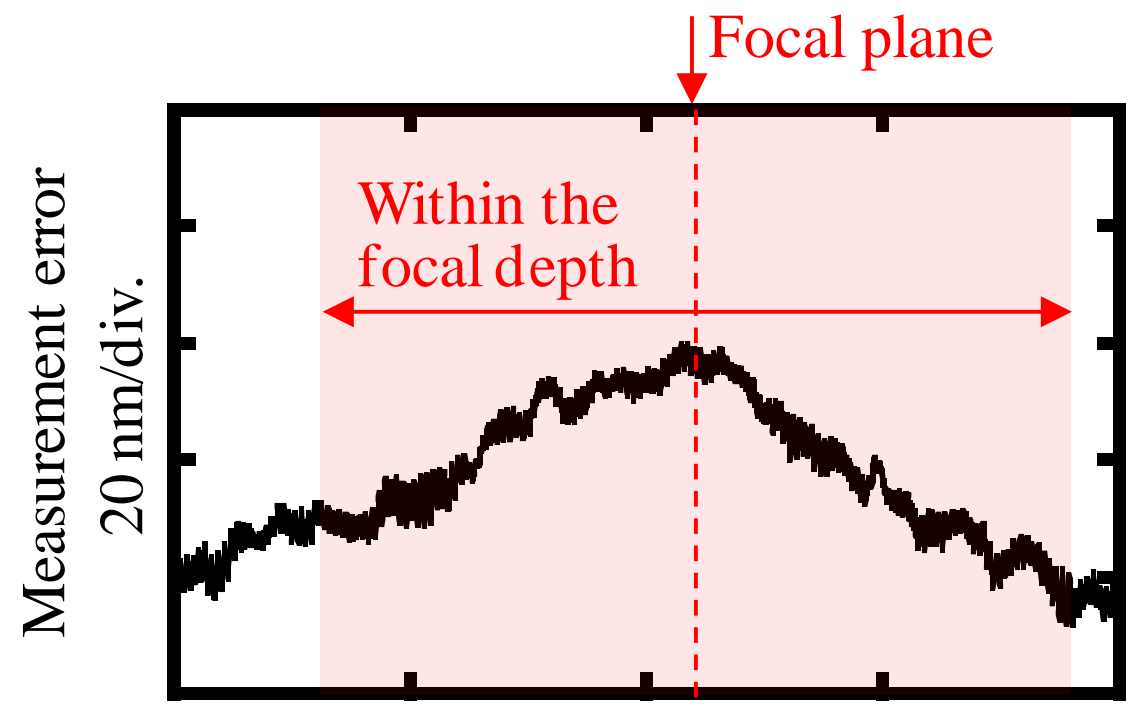

Displacement along the optical axis

$1 \mu \mathrm{m} / \mathrm{div}$.

Fig. 10 Measurement error due to the positioning error of the tool edge in the $Z$-direction 


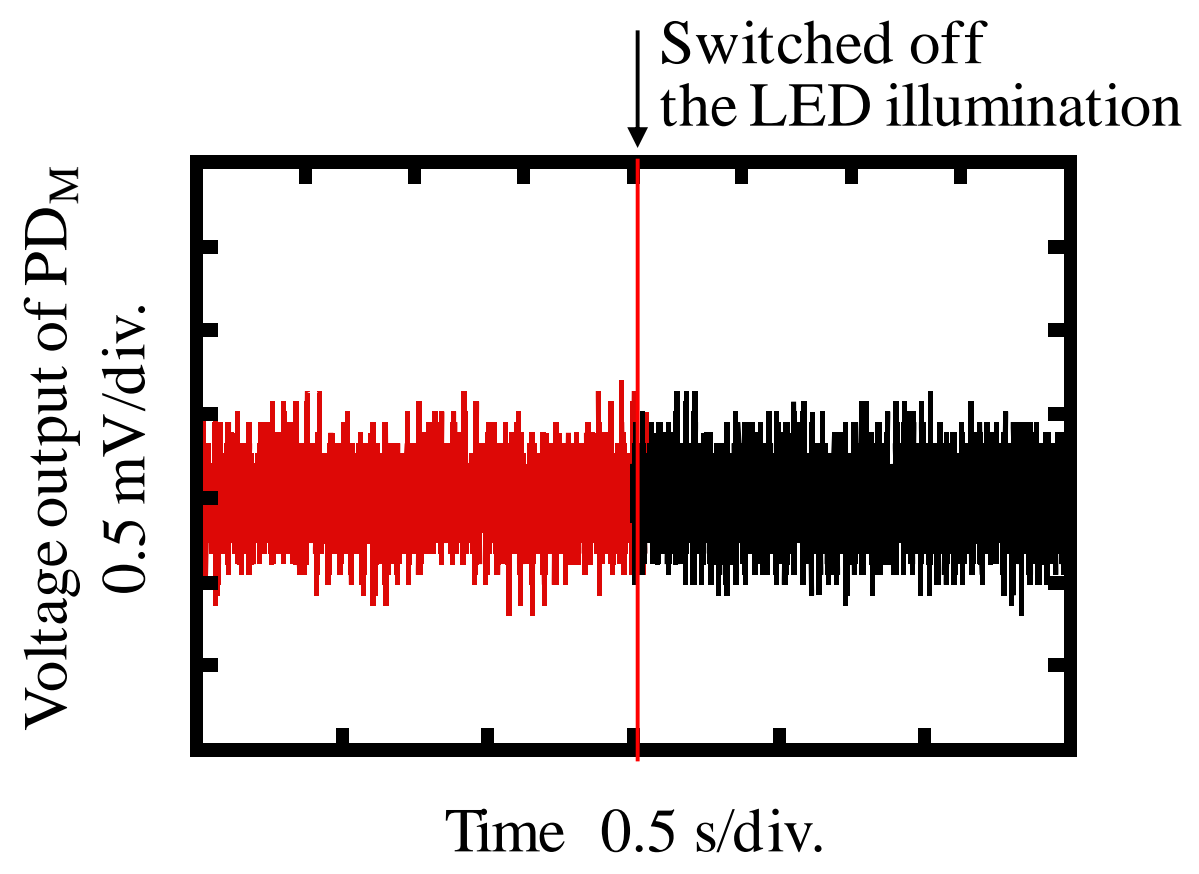

Fig. 11 Variation of the voltage output waveform from the photodiode $\mathrm{PD}_{\mathrm{M}}$ at the switching of the LED illumination 


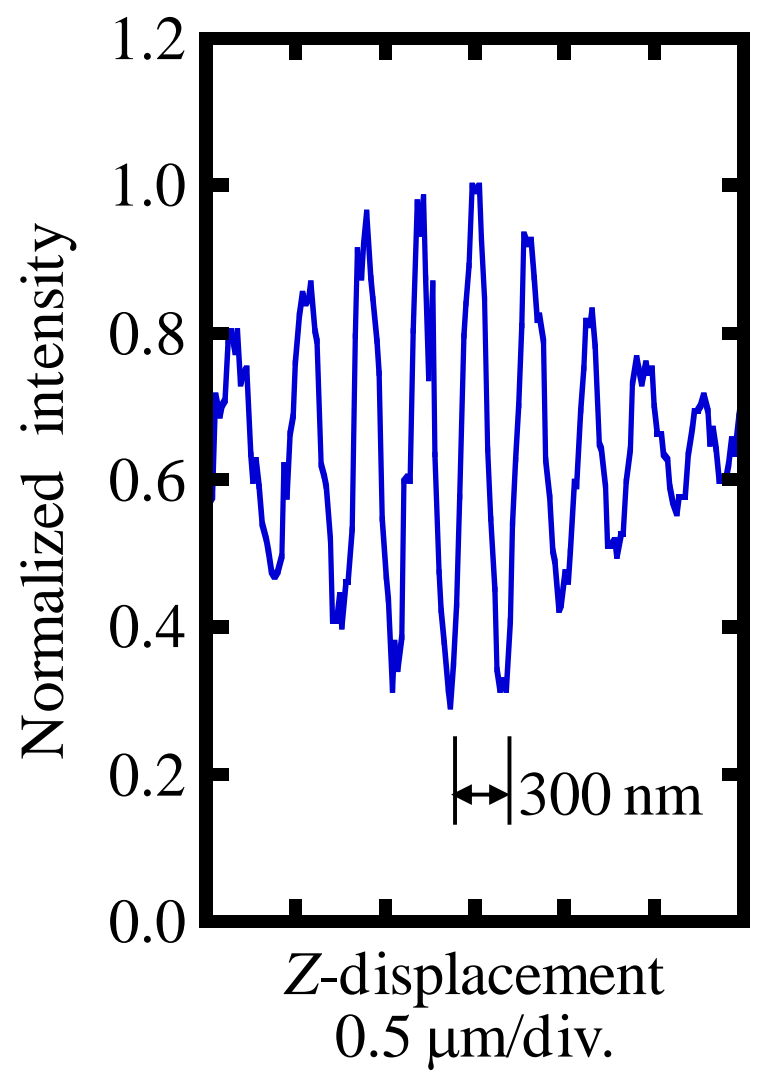

(a)

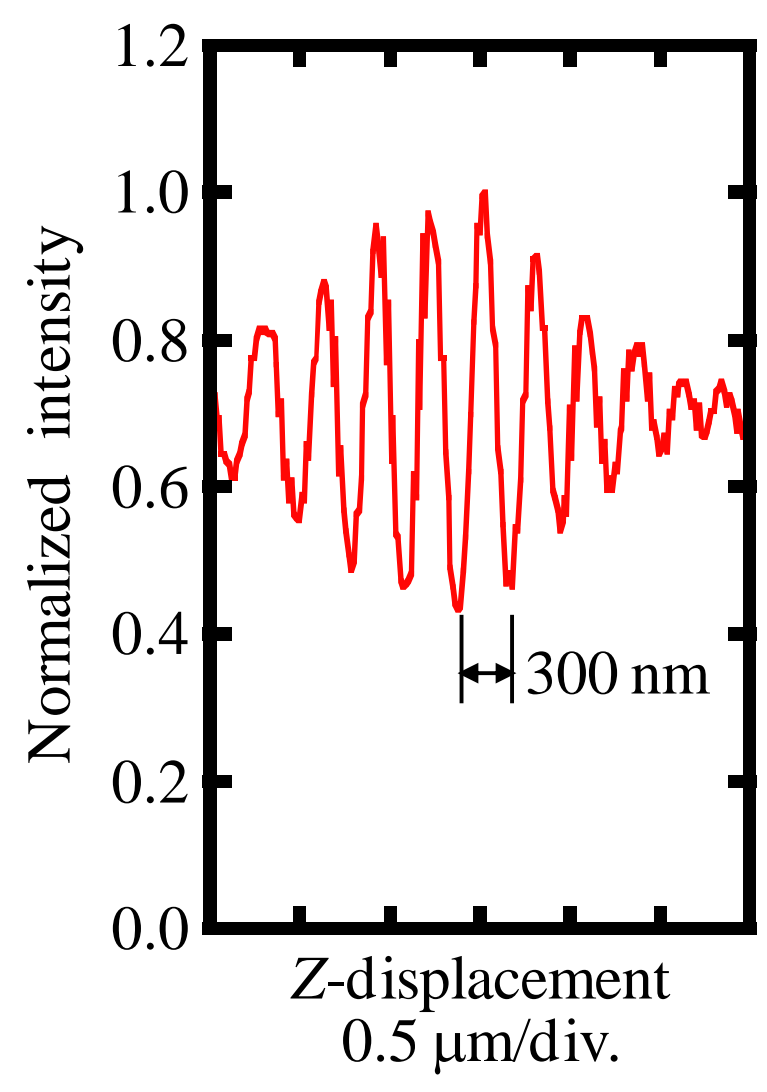

(b)

(a) Interference signal measured on the flat mirror surface

(b) Interference signal measured on the diamond tool face

Fig. 12 Measured interference signals 


\section{CCD image}

Rake face

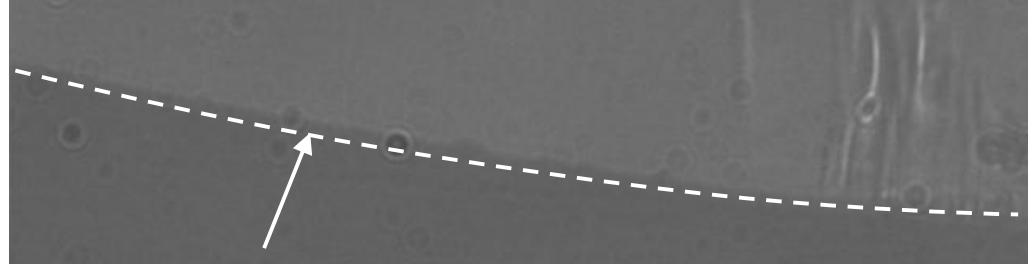

Tool edge

(a) CCD image

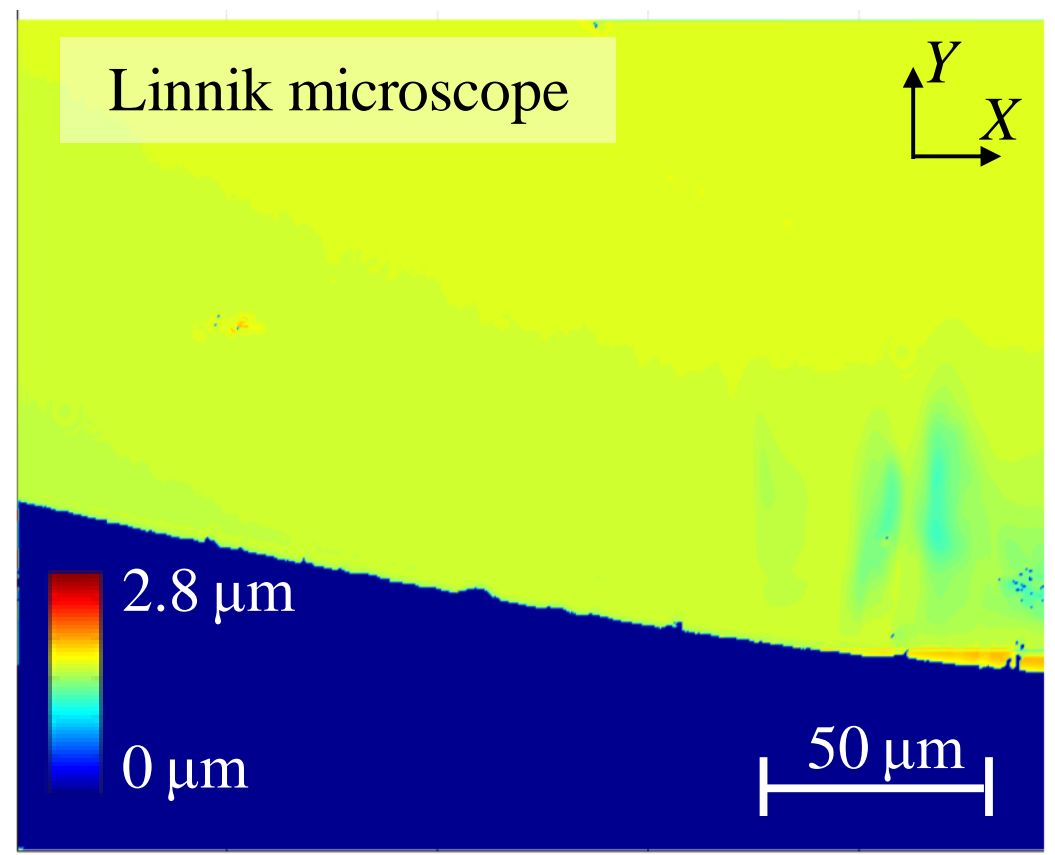

(b) Profile measured by the Linnik microscope component

Fig. 13 Measured tool rake face 


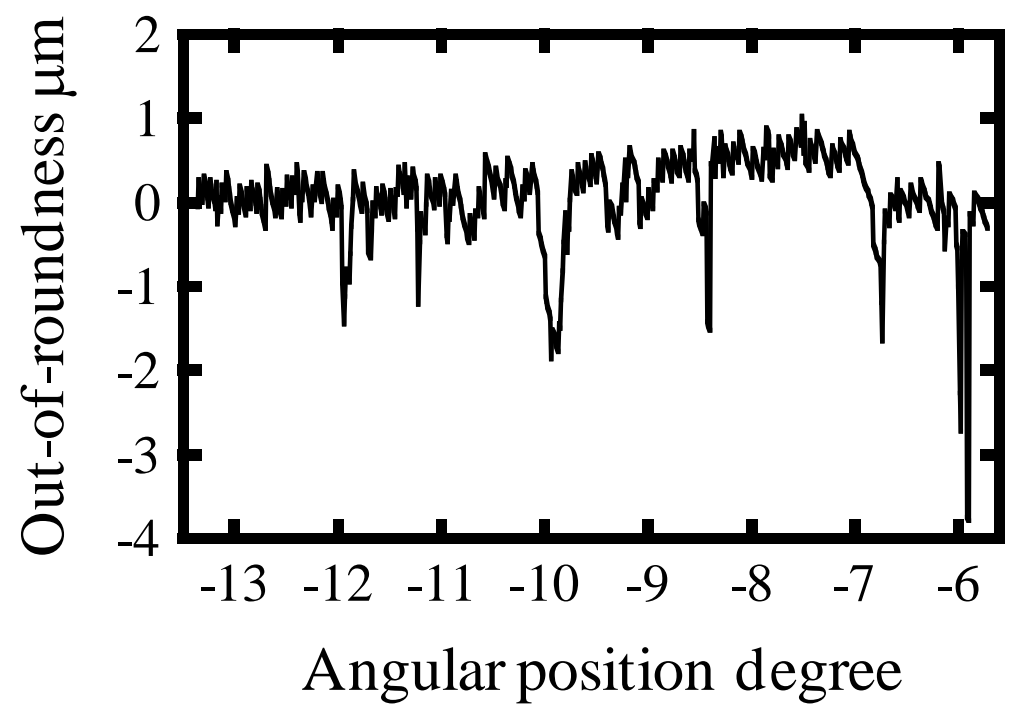

Fig. 14 Tool edge contour 


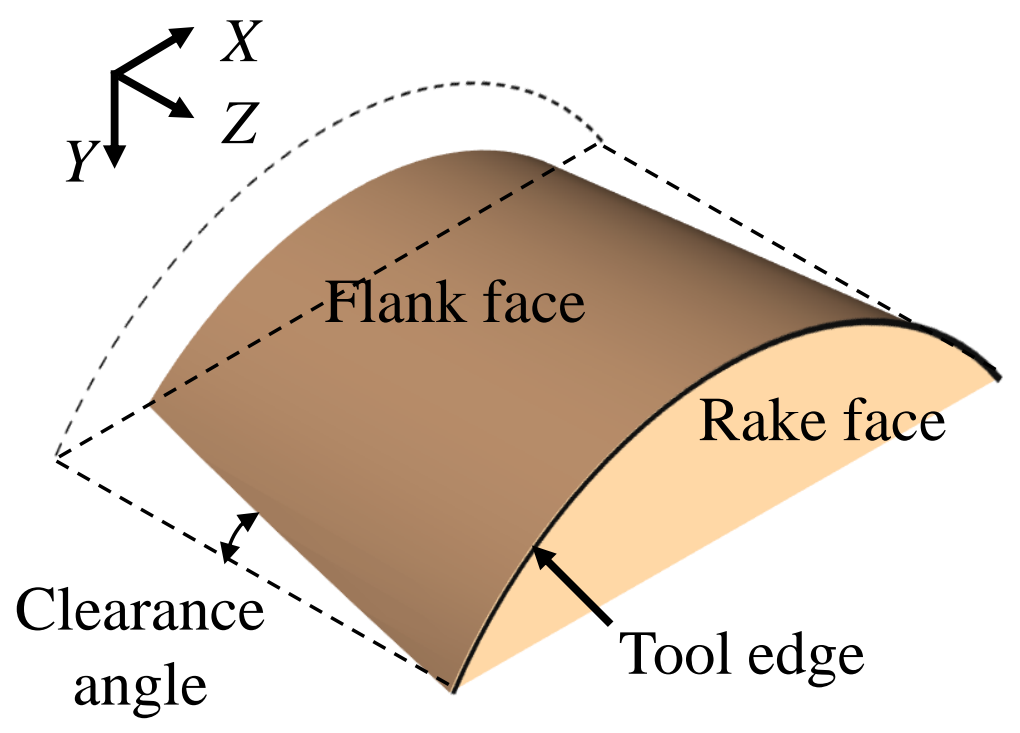

(a) A schematic of a flank face
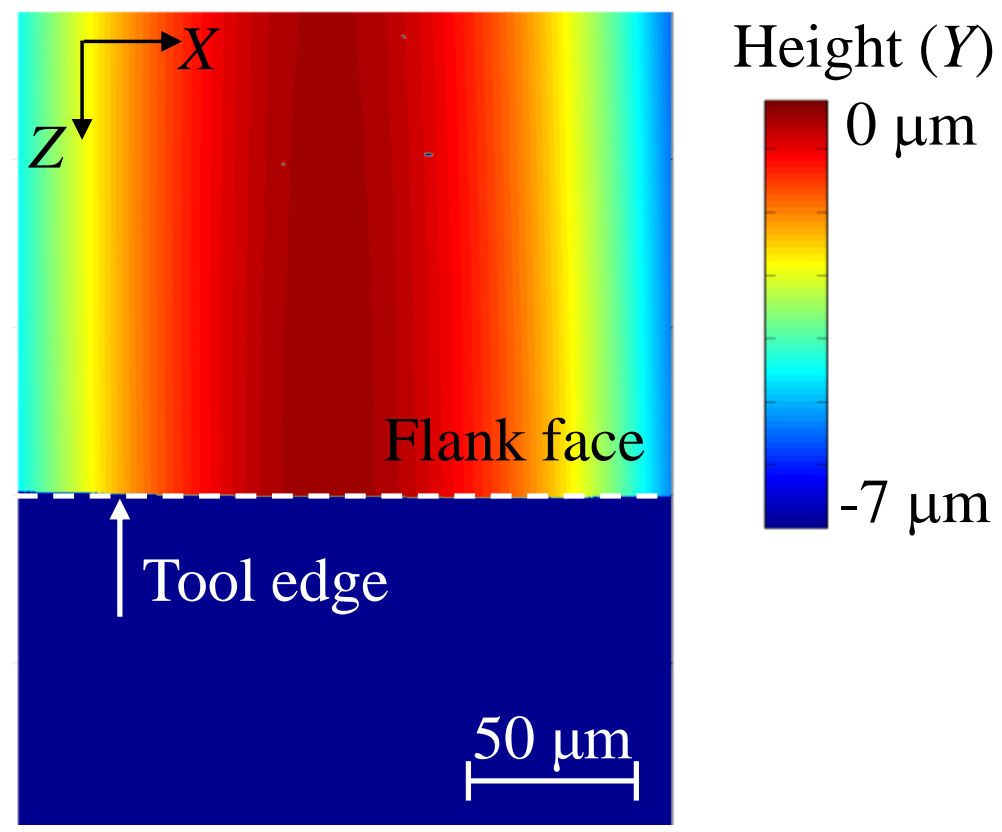

(b) Measured flank face

Fig. 15 Tool flank face measured by the developed instrument 